Daniel Sullivan, OMS III and McKennan Thurston, MD*

\title{
Acute fracture of extensive Achilles tendon calcific tendinopathy
}

https://doi.org/10.1515/jom-2020-0309

Received December 6, 2020; accepted January 26, 2021;

published online March 11, 2021

In May 2020, a 72 year old man presented to an outpatient orthopedic sports medicine clinic with a 3 week history of right ankle pain that began while mowing his lawn. One week after the onset of pain, the patient felt a "pop" and experienced subsequent bruising, swelling, and tenderness to his right lower leg. Walking was limited to short strides. At 2 years old, he underwent right lower leg surgery to correct in-toeing but had been asymptomatic since. Upon presentation to the orthopedic sports medicine clinic, the patient's Achilles tendon had an indurated, "woody" feel. Full range of motion was intact, and a Thompson test was negative. An Achilles tendon total rupture score was not taken. The contralateral leg was unremarkable. Laboratory tests were not performed upon initial presentation but were unremarkable several months later. In-office ultrasound revealed a linear hyperechoic signal with a segment of discontinuity (Image A, green arrow). Plain radiographs and magnetic resonance imaging (MRI) demonstrated ossification of the Achilles tendon with a fracture of the ossified mass (Image B, red arrow; Image $\mathbf{C}$, blue arrow). The patient agreed to a trial of nonsurgical treatment involving home exercises for range of motion, nonsteroidal anti inflammatory drugs (NSAIDs), and activity modification. Conservative treatment resulted in worse range of motion at the first follow up visit. The patient was then immobilized with a cast for 4 weeks and underwent physical therapy for several weeks thereafter. Five months after the inciting event, the patient reported no pain and full functionality.

\footnotetext{
*Corresponding author: McKennan Thurston, MD, Rocky Vista University College of Osteopathic Medicine, 1730 E 3100 N, Ivins, UT, 84040-2407, USA, E-mail: mckennan.thurston@gmail.com Daniel Sullivan, OMS III, Rocky Vista University College of Osteopathic Medicine, Ivins, UT, USA
}
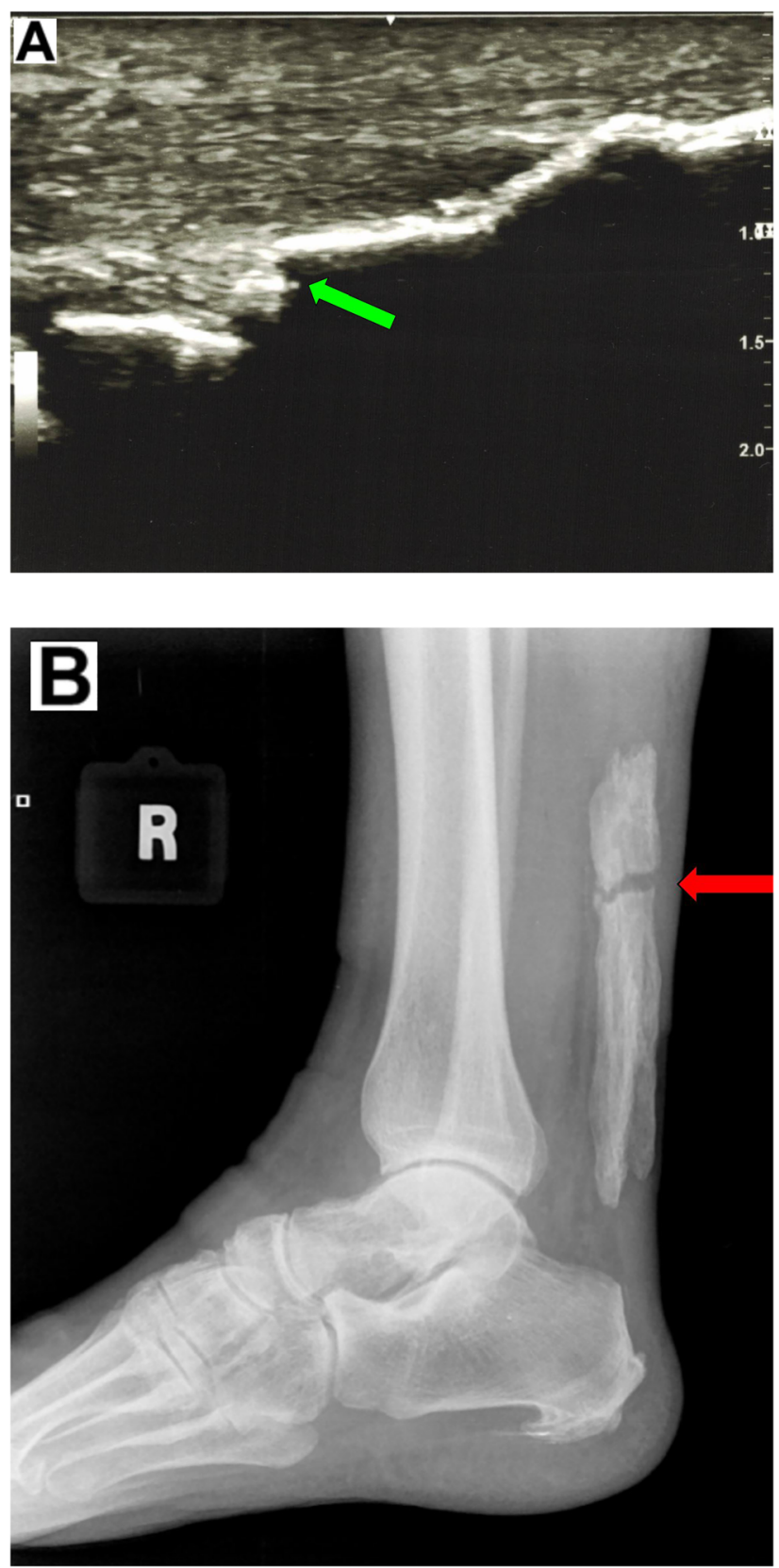


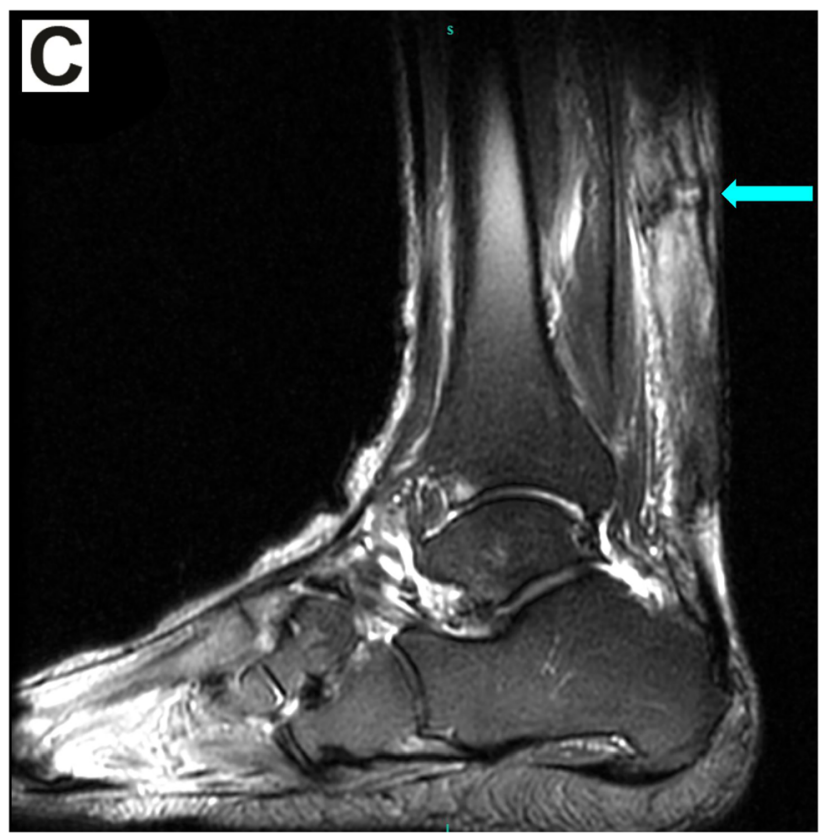

Extensive calcification of the Achilles tendon with a subsequent fracture is exceedingly rare $[1,2]$. As with this case, previous reports have described patients who experienced acute trauma affecting the Achilles tendon decades prior [2-5]. A fracture of the calcified tendon may or may not result in a complete tendon rupture. Conservative and surgical treatments have both been reported. Conservative interventions include ice and heat application, range of motion exercises, oral and topical NSAIDs, orthotics, and physical therapy [6]. Surgical interventions include replacing the tendon with an autograft from the tensor fasciae latae [7], flexor hallucis longus tendon [2], or hamstring tendon [8].

Research funding: None declared.
Author contributions: Both authors provided substantial contributions to conception and design, acquisition of data, or analysis and interpretation of data; both authors drafted the article or revised it critically for important intellectual content; both authors gave final approval of the version of the article to be published; and both authors agree to be accountable for all aspects of the work in ensuring that questions related to the accuracy or integrity of any part of the work are appropriately investigated and resolved.

Competing interests: Authors state no conflict of interest.

\section{References}

1. Arora AJ, Arora R. Ossification of the bilateral Achilles tendon: a rare entity. Acta Radiol Open 2015;4:205846011559918.

2. Battaglia TC, Chandler JT. Ossific tendonitis of the Achilles with tendon fracture. Orthopedics 2006;29:453-5.

3. Cortbaoui C, Matta J, Elkattah R. Could ossification of the Achilles tendon have a hereditary component? Case Rep Orthop 2013;2013. https://doi.org/10.1155/2013/539740.

4. Majeed H, Deall C, Mann A, Mcbride DJ. Multiple intratendinous ossified deposits of the Achilles tendon: case report of an unusual pattern of ossification. Foot Ankle Surg 2015;21. https://doi.org/ 10.1016/j.fas.2014.12.001.

5. Manfreda F, Ceccarini P, Corzani M, Petruccelli R, Antinolfi P, Rinonapoli G, et al. A silent massive ossification of Achilles tendon as a suspected rare late effect of surgery for club foot. SAGE Open Med Case Rep 2018;6. https://doi.org/10.1177/ $2050313 \times 18775587$.

6. Maffulli N, Longo UG, Kadakia A, Spiezia F. Achilles tendinopathy. Foot Ankle Surg 2020;26:240-9.

7. Gendera H, Lambers-Heerspink F, Bruls V, Drees M. Extensive Achilles tendon ossification: repair using a fascia lata graft. Foot 2020;43:101663.

8. Ishikura H, Fukui N, Takamure H, Ohashi S, Iwasawa M, Takagi K, et al. Successful treatment of a fracture of a huge Achilles tendon ossification with autologous hamstring tendon graft and gastrocnemius fascia flap: a case report. BMC Muscoskelet Disord 2015;16. https://doi.org/10.1186/s12891-015-0821-x. 